\title{
ARTICLES
}

\section{Inherited Motor Neuron Disease in Domestic Cats: A Model of Spinal Muscular Atrophy}

\author{
QIANCHUAN HE, CHARLES LOWRIE, G. DIANE SHELTON, RUDY J. CASTELLANI, \\ MARILYN MENOTTI-RAYMOND, WILLIAM MURPHY, STEPHEN J. O’BRIEN, \\ WILLIAM F. SWANSON, AND JOHN C. FYFE
}

\begin{abstract}
Laboratory of Comparative Medical Genetics [Q.H., J.C.F.], Department of Microbiology and Molecular Genetics, and Department of Small Animal Clinical Sciences [C.L., J.C.F.], College of Veterinary Medicine, Michigan State University, East Lansing, MI 48824; Comparative Neuromuscular Laboratory [G.D.S.], Department of Pathology, School of Medicine, University of California, San Diego, La Jolla, CA 92093; Division of Neuropathology [R.J.C.], College of Human Medicine, Michigan State University, East Lansing, MI 48824; Laboratory of Genomic Diversity [M.M.-R., W.M., S.J.O.], NCI-Frederick, Frederick, MD 21702; and Center for Research of Endangered Wildlife [W.F.S.], Cincinnati Zoo \& Botanical
\end{abstract}

Garden, Cincinnati, $\mathrm{OH} 45220$

\begin{abstract}
ABS
Juvenile-onset spinal muscular atrophy was observed in an
extended family of purebred domestic cats as a fully penetrant,
simple autosomal recessive trait. Affected kittens exhibited
tremor, proximal muscle weakness, and muscle atrophy begin-
ning at $\sim 4$ mo of age. Apparent loss of function was rapid
initially but progressed slowly after $7-8$ mo of age, and variably
disabled cats lived for at least 8 y. Electromyography and
microscopic examination of muscle and nerve biopsies were
consistent with denervation atrophy as a result of a central lesion.
There was astrogliosis and dramatic loss of motor neurons in
ventral but not dorsal horn gray matter of spinal cord and loss of
axons in ventral horn nerve roots. These phenotypic findings
were similar to mild forms (type III) of spinal muscular atrophy
\end{abstract}
in humans caused by survival of motor neuron mutations, but molecular analysis excluded feline survival of motor neuron as the disease gene in this family. A breeding colony has been established for further investigation of this naturally occurring large-animal model of inherited motor neuron disease. (Pediatr Res 57: 324-330, 2005)
Abbreviations
RT-PCR, reverse transcription-PCR
SMA, spinal muscular atrophy
$S M N$, telomeric survival of motor neuron gene
5qSMA, SMA due to mutations of $S M N$

The spinal muscular atrophies (SMAs) are a group of inherited disorders characterized by neurogenic muscle atrophy and weakness as a result of spinal lower motor neuron degeneration with sparing of upper motor neurons (1). They compose a large category of inherited disease, but the molecular basis has been established for only a few. The SMAs are clinically categorized by pattern of inheritance and distribution of muscle

Received May 20, 2004; accepted August 25, 2005.

Correspondence: John C. Fyfe, D.V.M., Ph.D., Laboratory of Comparative Medical Genetics, 2209 Biomedical Physical Sciences, Michigan State University, East Lansing, MI 48824; e-mail: fyfe@cvm.msu.edu.

This work was supported by grants from the National Institute of Child Health and Human Development (HD 39888), the National Institute of Research Resources (RR 15388), and the Companion Animal Fund of Michigan State University College of Veterinary Medicine.

DOI: 10.1203/01.PDR.0000153625.46892.6F atrophy (1,2). Recessive proximal SMA has been subdivided further into three types on the basis of age of onset, survival time, and clinical severity $(1,3,4)$.

Type I, Werdnig-Hoffman disease, is characterized by fetal or perinatal onset and death by a few months of age and is the most common autosomal recessive disease that is lethal to infants (5). Type II is an intermediate form with slightly later onset, a more chronic course, and death by a few years of age. Type III, Kugelberg-Welander disease, has onset between 2 and $17 \mathrm{y}$ of age and is compatible with life into the third to sixth decades. Longer survival and weight bearing in type III patients correlate with progression to distal muscle involvement and from strictly neurogenic atrophy to variable changes that may seem myopathic. In the intermediate and mild clinical forms of SMA, patients typically experience rapid decline of 
muscle strength at the onset of disease but retain a steady level for years or decades thereafter. This observation is compatible with rapid loss of motor neurons early in the disease followed by increasing stability of surviving neurons, as is also suggested by mathematical modeling of motor neuron degeneration in a variety of neuronal death disorders and models (6). The characteristic pathologic features of all SMA types at the time of diagnosis are low numbers of surviving spinal lower motor neurons and denervation atrophy of skeletal muscle $(2,5,7)$.

The most common SMA ( 1:6,000-10,000 births; herein called 5qSMA) is an autosomal recessive disorder characterized by symmetrical atrophy and weakness of proximal muscles that is attributable to mutations of the telomeric copy of the survival of motor neuron gene (SMN1) located on chromosome $5 q 12-q 13(1,3)$. There is a relatively high mutation rate at this locus in humans as a result of an accident of evolution that produced an inverted duplication of $500 \mathrm{kbp}$ and predisposes SMN1 to deletion and gene conversion events (8). Whereas most cases of SMA types I-III are attributable to various mutations of the SMN1 locus, in some type I and at least $5 \%$ of type II and type III patients, families do not show linkage to the SMN1 locus, indicating that there is locus heterogeneity in human SMA (9). This report describes the clinical, pathologic, and genetic features of juvenile-onset SMA in a family of purebred domestic cats that is phenotypically homologous to the mild forms of SMA in humans but is not caused by mutation of $S M N$.

\section{METHODS}

Animals. Privately owned purebred Maine coon cats, including 12 affected cats, six clinically normal littermates, and four of their parents, were examined. All clinical examinations and biopsies were performed at the Veterinary Teaching Hospital of the College of Veterinary Medicine, Michigan State University, or at the Hidden Valley Animal Hospital, McMurray, PA, adhering to the highest standards of veterinary practice and with informed consent of owners. Humane euthanasia was performed according to protocols approved by the All University Committee for Animal Use and Care of Michigan State University and conforming to National Institutes of Health guidelines.

Electromyography and nerve conduction velocity. Electromyographic evaluation was performed on five cats under inhalation anesthesia. Parameters assessed included evidence and severity of increased insertional activity as well as the presence of abnormal spontaneous activity (fibrillation potentials, positive sharp waves, and complex repetitive discharges). Motor nerve conduction velocity was determined in sciatic nerves by dividing the distance between proximal and distal stimulation sites by the difference in latency of the corresponding compound muscle action potentials recorded from the plantar interosseous muscles after supramaximal stimulation.

Histopathology and electron microscopy. Tissues for light microscopy were fixed by immersion in $1.25 \mathrm{M}$ neutral buffered formaldehyde (10\%), paraffin or plastic (glycol methacrylate) embedded, and sectioned. In some cats, rapid fixation of the CNS was accomplished by $10 \%$ neutral buffered formaldehyde perfusion via the left cardiac ventricle. Routine stains included hematoxylin-eosin, Luxol-fast blue, periodic acid-Schiff, toluidine blue, and cresyl violet. Unfixed muscle specimens were flash-frozen in isopentane precooled in liquid nitrogen and kept frozen at $-70 \mathrm{C}$ before sectioning. A standard panel of histochemical stains and enzymatic reactions including hematoxylin-eosin, modified Gomori trichrome, ATPases at $\mathrm{pH} 9.8$ and 4.3, esterase, NADH-TR, periodic acid-Schiff, and acid phosphatase were performed on 10- $\mu \mathrm{m}$ sections (10). Paraffin-embedded sections of spinal cord were immunostained by sequential incubation with polyclonal rabbit antibovine glial fibrillary acidic protein antiserum (Dako Corp., Carpinteria, CA), biotinylated anti-rabbit $\operatorname{IgG}(\mathrm{H}+\mathrm{L})$, avidin-horseradish peroxidase conjugate, and Nova Red peroxidase substrate (Vector Laboratories, Burlingame, CA). Spinal cord white and gray matter volumes of $\mathrm{C}_{6}$ and $\mathrm{S}_{1}$ segments were evaluated by point counting $\left(0.53-\mathrm{mm}^{2}\right.$ cell area) on every other of 20 serial
5- $\mu \mathrm{m}$ sections (11). In the same sections, all neurons in which the nucleolus was visible were counted and categorized as having cell body diameter $>$ or $<50 \mu \mathrm{m}$. Tissue for electron microscopy was fixed by overnight immersion in phosphate-buffered $0.4 \mathrm{M}(4 \%)$ of glutaraldehyde, postfixed in $40 \mathrm{mM}(1 \%)$ of osmium tetroxide, dehydrated in graded alcohols, and embedded in Poly/Bed 812-Araldite resin. Sections were cut, stained sequentially in uranyl acetate and citrate-buffered lead nitrate, and examined on a Philips 301 transmission electron microscope. Spinal nerve roots for teased-fiber preparation were fixed, postfixed in osmium tetroxide, dehydrated, teased, and embedded in resin as above but on glass slides for light microscopy.

Library screening. A portion of feline $S M N$ cDNA was amplified by oligo dT primed reverse transcription-PCR (RT-PCR) as described (12), using cat liver RNA and the PCR primers 5'-GATGATTCTGACATTTGGGATGATAC-3' and 5'-GCATCATCACGCGAATCTGGAC-3'. The PCR step was carried out in $50-\mu \mathrm{L}$ reactions that contained $1.5 \mathrm{mM}$ of $\mathrm{MgCl}_{2}$ for 35 cycles at $95^{\circ} \mathrm{C}$ for $20 \mathrm{~s}$, $57^{\circ} \mathrm{C}$ for $30 \mathrm{~s}$, and $72^{\circ} \mathrm{C}$ for $160 \mathrm{~s}$. The product was sequenced by automated dideoxy nucleotide chain termination chemistry in the University of Michigan DNA Sequencing Core Laboratory. The primers 5'-TTGGTCAGAAGACGGCTGCGTT-3' and 5'-CCAGCATGATAGGAAGTGTGGTGG-3' designed from the feline $S M N$ cDNA sequence were used in a pooled PCR-based method (13) to screen $3 \times 10^{7}$ pfu of a cat testis cDNA bacteriophage $\lambda$ library (14). After plaque purification, clones were rescued into the phagemid pBluescript (Stratagene, La Jolla, CA) and sequenced completely on both strands. Sequences were assembled and analyzed using the Lasergene software (DNAstar, Madison, WI). The fulllength feline $S M N$ cDNA sequence was submitted to the EMBL/GenBank Data Libraries (accession no. AY094503)

Transcript analysis. Feline SMN mRNA transcripts were amplified by RTPCR from cat liver RNA, as above. The PCR primers were 5'-TGTACCGGGCAGTAGGCT-3' in the 5' untranslated region (UTR) and 5'-TTCAGTCGATATTTTCACAGATTG-3 in the $3^{\prime}$ UTR, and the products were sequenced. For analyzing a sequence variation in the noncoding exon 8, RT-PCR was performed as above but with the primers 5'-AAAGCCTGGTCTAAAGTTCAATGG-3' and 5'-AGGGCAATGCAGATGGCTATG-3', the products were digested with the restriction endonuclease Xmn I, and restriction fragments were separated by electrophoresis on $1 \%$ agarose gels. PCR products were also cloned into pCR-XL-TOPO (Invitrogen/Life Technologies, Carlsbad, CA), and multiple clones were sequenced.

Southern blotting. A 3.3-kbp PCR product of the $S M N$ gene was amplified from genomic DNA using the exon 3 and exon 5 primers 5'-TTGGTCAGAAGACGGCTGCGTT-3' and 5'-CCAGCATGATAGGAAGTGTGGTGG-3', respectively, and sequenced. An EcoRV digestion fragment of the PCR product spanning part of exon 3 , intron 3 , exon 4 , and part of intron 4 was used as hybridization probe. Enzymes for restriction digests that did not have a recognition site in the probe sequence were chosen. Ten micrograms of cat genomic DNA was digested with each of the restriction endonucleases $B a m \mathrm{H}$ I, HindIII, XbaI, StuI, BglII, NotI, XhoI, and SacI. Digestion fragments were separated by electrophoresis on a $0.8 \%$ agarose gels, transferred to a nylon membrane, and hybridized with random-primed labeled probe DNA by standard methods (15).

Linkage analysis. Genomic DNA of cats in the SMA family was PCR amplified with primers 5'-AGACGCTTGACTGATTGACC-3' and 5'TCTCTGCCCCTTCCCCACTTA-3' flanking a GTTT tandem repeat sequence variation in intron 4 of SMN. PCR products were separated on $12 \%$ polyacrylamide gels and stained with ethidium bromide. PCR-based radiation hybrid mapping of feline SMN was performed on a 93-hybrid 5000-rad feline wholegenome radiation hybrid panel as described $(14,16)$.

\section{RESULTS}

Inheritance of feline SMA. Figure 1 shows a family pedigree of cats exhibiting lower motor neuron loss and SMA segregating as an autosomal recessive trait. Thirteen affected cats, seven male and six female, produced in three privately owned catteries, were examined and/or followed. In the four pairings that produced affected kittens, one of two, three of eight, three of six, and six of 25 total offspring were affected. These results did not differ significantly from the fraction of affected offspring (0.25) and even gender distribution expected under the hypothesis of simple autosomal recessive inheritance with full penetrance $\left(\chi^{2}=0.4, d f=1, p=0.53\right)$. Consistent with the expectation that the disease allele was homozygous by descent in each affected cat, all parents of affected cats were 


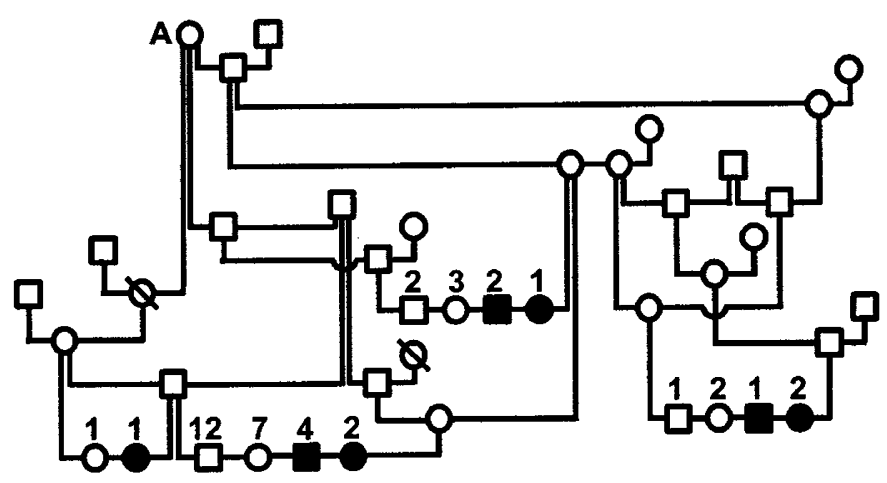

Figure 1. Family pedigree of cats that exhibit lower motor neuron loss and SMA. Squares indicate males, circles indicate females, open symbols indicate clinically normal cats, and filled symbols indicated affected cats. Numerals above certain symbols indicate the number of offspring of the indicated gender and phenotype obtained in multiple litters from the indicated mating. The female indicated by $\mathbf{A}$ is an ancestor common to every parent of an affected cat, and all cats in this family were purebred Maine coon cats.

clinically normal and shared at least one common ancestor (cat A) within five generations.

Clinical findings. Affected cats were examined at a variety of ages from $15 \mathrm{wk}$ to $6.5 \mathrm{y}$, but in each instance, onset of clinical signs was noted between 13 and 17 wk of age. Initial abnormalities were abnormal gait and a fine muscle tremor, particularly over the hindquarters. Weakness was evident in all but more so in pelvic limbs. At all examinations, the cats were alert and responsive. There were no deficits of cranial nerves or spinal reflexes. Postural and proprioceptive deficits were not observed except where attributable to muscle weakness. By 5 mo of age, all affected kittens walked with wide-based forelimbs and a side-to-side sway of the hindquarters. They walked and stood with tibiotarsal joints in the normally elevated position, suggesting that gastrocnemius muscle strength was maintained. Weakness of proximal hindlimb muscles was expressed in the characteristic stance of tibiotarsal joints nearly touching and hind feet rotated laterally to an angle of $30-40^{\circ}$ from the midline, apparently taken to gain stability. Muscle atrophy was apparent by 4-5 mo of age in proximal limb muscles, particularly of the hindlimbs. Shallow tachypneic breathing was observed in some affected cats, and all often sat or lay down when walking across a room. There was no radiographic evidence of diaphragmatic eventration or skeletal abnormalities.

After an initial 4-8 mo of disease progression, the ongoing loss of muscle function seemed to slow and affected cats reached a clinical plateau of variable muscle atrophy, weakness, and mobility. Two affected female cats were ambulatory and weight bearing on all four limbs at 5 and 6 y of age despite extensive muscle atrophy. The oldest and most severely affected cat, a male, had previously moved with a "bunny hop" type of gait in the hindquarters, but by $2.5 \mathrm{y}$ of age, the cat had hindlimb flaccid paralysis. On rare occasion, he scaled a pair of carpeted steps by pulling himself up with his fore limbs. At the most recent examination, he was $6.5 \mathrm{y}$ of age, and all limb and trunk muscles were severely atrophied. The forelimbs still supported weight, but hindlimb muscles were flaccid. There was no evidence of muscle contracture or joint fixation. Al- though apparently sensitive to the stimulus, he was incapable of withdrawing the hindlimbs in response to a toe pinch. Despite these disabilities, he continued to eat well and remained alert to external stimuli such as the play of other cats in the household.

The only abnormality revealed in complete blood counts, serum biochemistries, and urinalyses was a 2- to 3-fold elevation of creatine phosphokinase activity (705-964 IU/L; normal range 83-317 IU/L), a finding that was inconsistent with inflammatory or dystrophic muscle disease. Urine screening (17) for abnormal metabolites revealed no abnormalities suggestive of major perturbations of amino acid or carbohydrate intermediate metabolism. Semen collected from an 18-mo-old affected cat showed no abnormalities, the collected sperm successfully produced in vitro embryos that developed into live offspring after embryo transfer, and another affected male produced live offspring through natural mating.

In affected cats that were examined at $15 \mathrm{wk}$ to $1.5 \mathrm{y}$ of age, electromyography (EMG) demonstrated fibrillation potentials, positive sharp waves, and increased insertional activity variably in multiple muscles of neck, truck, and limbs, consistent with a denervation type of muscle pathology. In every affected cat examined, pelvic and pectoral girdle muscles and thoracolumbar epaxial muscles exhibited abnormal electrical activity. Abnormalities were most often observed in lumbar epaxial muscles, caudal muscles of the thigh, and the cranial tibialis muscle, the last being flexors of the femorotibial and tibiotarsal joints, respectively. In the youngest cats with recent onset of signs, EMG abnormalities were distributed more generally than in older affected cats, including all epaxial muscles and many cranial and caudal muscles of proximal and distal portions of all four limbs. Motor nerve conduction velocities of sciatic nerves were normal.

Histopathology. Hindlimb muscle specimens of all cats biopsied were consistent with denervation. There was variability in myofiber size with numerous singular atrophic fibers having angular shapes and small groups of atrophic fibers (Fig. $2 A)$. The atrophic fibers were of both fiber types with general preservation of myofiber-type mosaicism (Fig. 2B). The muscle lesions were more generalized in older cats with more chronic disease in which larger groups of atrophic fibers and mild fiber-type grouping was suggestive of axon sprouting and renervation by surviving neurons. Neither inflammatory nor dystrophic changes were noted in any of the muscle specimens examined. Peripheral nerve biopsy specimens revealed a fascicular pattern of mild nerve fiber loss with occasional small, thinly myelinated nerve fibers. Intramuscular nerve branches showed variable depletion of myelinated nerve fibers. The biopsy findings were most consistent with denervation resulting from spinal motor neuron degeneration, as opposed to a motor neuropathy or primary myopathy.

Seven affected cats, males at 3.5, 5.5, 12, and 18 mo and females at 5.5, 8, and 60 mo of age, were donated for euthanasia and histopathologic examination. Lesions were observed only in spinal cord, peripheral nerves, and skeletal muscle and were consistent with the findings in muscle and nerve biopsies described above. No abnormalities were observed in spinal cord white matter tracts, taking particular note of the lateral 

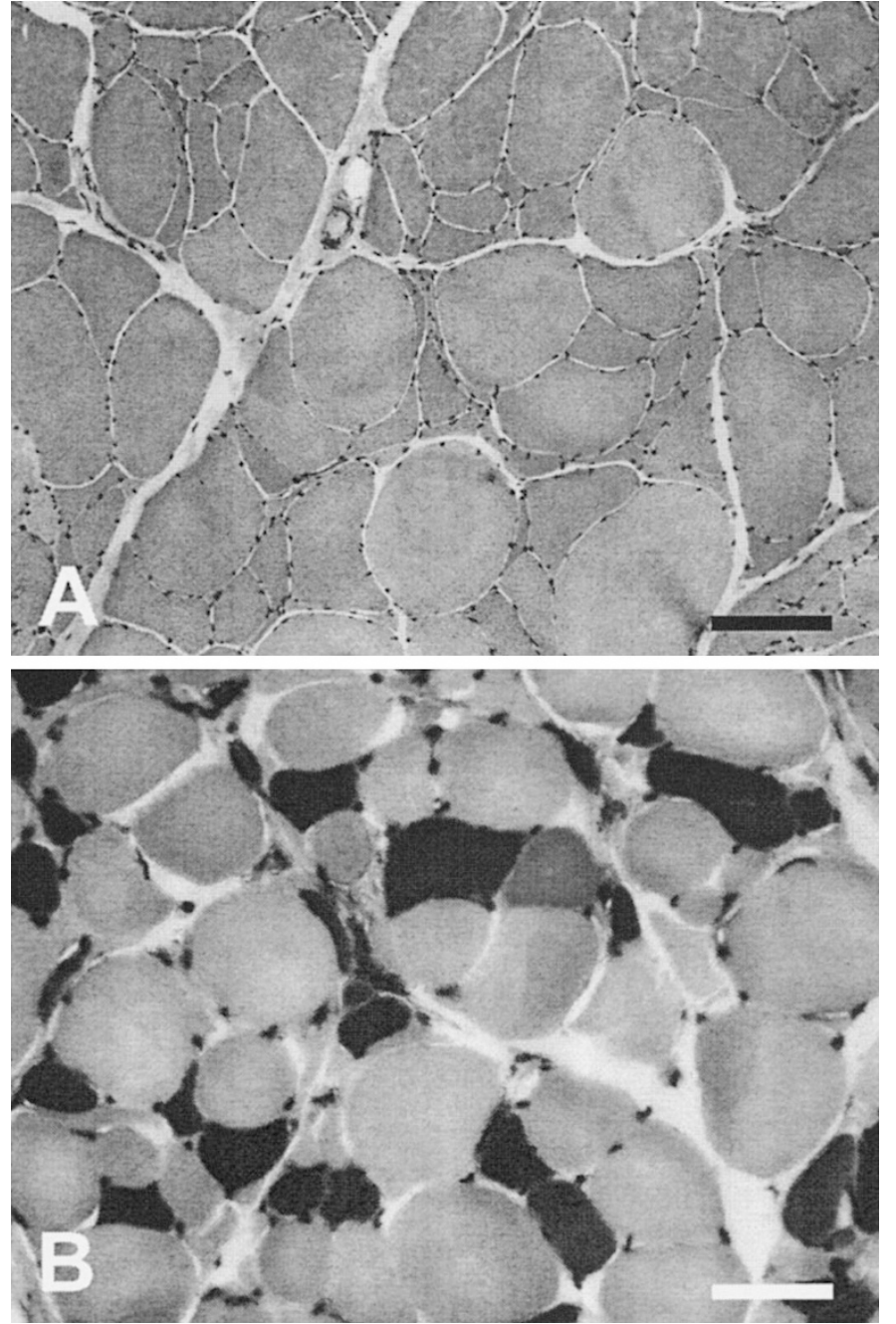

Figure 2. Photomicrographs of hindlimb skeletal muscle of a cat exhibiting clinical signs of SMA. Pathologic changes in fresh-frozen muscle biopsy specimens $(A ; \mathrm{H} \& \mathrm{E}$ stain) included variation in myofiber size with scattered singular angular atrophied fibers and groups of atrophic fibers. The atrophic fibers were of both fiber types without fiber-type grouping ( $B$; myofibrillar ATPase reaction, $\mathrm{pH} 4.3$ ). Bars $=50 \mu \mathrm{m}$.

corticospinal tracts, and there were no definitive pathologic changes present in the brainstem or more rostral CNS by light microscopy. No inclusions of abnormal storage material were seen in any part of the CNS. In spinal cord gray matter of the youngest cat, many large neurons of the ventral horns were generally dark staining with toluidine blue and had pale cytoplasmic staining with fragmented Nissl substance in cresyl violet. Transmission electronmicrographs of these cells revealed misshapen and indented nuclei, dilated mitochondria and rough endoplasmic reticulum (RER), and separation of RER by finely granular material containing islands of aggregated filaments (Fig. 3). These cells were not observed in an age-matched control cat or in affected cats that were $24 \mathrm{wk}$ of age or older, suggesting that early degenerative changes progressed to motor neuron loss (see below).

In affected cats that were $24 \mathrm{wk}$ of age and older, there was an obvious paucity of large ventral horn neurons (Fig. 4A-D). Some of the remaining motor neurons had a normal appearance, but others exhibited pathologic changes that were visible

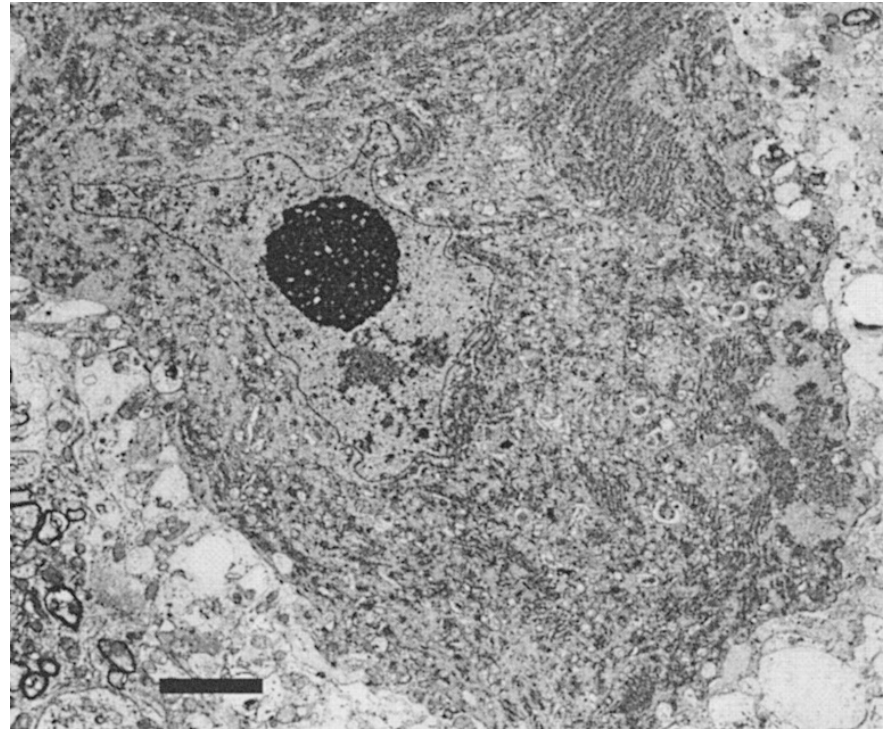

Figure 3. Electronmicrograph of a ventral horn motor neuron perikaryon from a 15-wk-old cat exhibiting clinical signs of SMA. Pathologic changes included irregular contour of the nuclear membrane, dilated mitochondria and endoplasmic reticulum, and highly fragmented Nissl substance. Bar $=5 \mu \mathrm{m}$.

by light microscopy, including cytoplasmic chromatolysis and eccentrically placed, pale staining, misshapen nuclei. Occasionally, gliotic figures suggestive of neuronophagia were observed.

Some cats were studied in greater detail. There was no significant difference in gray or white matter volumes in cervical, thoracic, lumbar, or sacral cord segments of two normal and two affected cats at 24 wk of age. However, counting indicated 18 - to 28 -fold reduction in neurons with perikaryon diameter of $>50 \mu \mathrm{m}$ in the spinal cord segments of affected cats. The large neurons composed only $0.25 \%$ (normal $=$ $5.5 \%$ ) of cervical and $0.3 \%$ (normal $=8 \%$ ) of sacral segment total neurons in the affected cats. Increased glial fibrillary acidic protein immunoreactivity and astrocyte hypertrophy observed in ventral but not dorsal horn gray matter of affected cat spinal cord suggested that the degenerative process was confined to ventral horn cells (Fig. $4 E$ and $F$ ).

Findings were similar in cross-sections of spinal cord of a 12-mo-old male and a 5-y-old female affected cat. In addition, in these cats, dorsal and ventral nerve root axons were counted and measured (Figs. 5 and 6). There was dramatic loss of large myelinated axons and replacement by endoneurial connective tissue observed in cross-sections of ventral but not dorsal nerve roots. In teased-fiber preparations, $\sim 10 \%$ of axons that remained in ventral nerve roots of the affected cats showed evidence of degeneration that was not observed in dorsal nerve roots or in control cat ventral or dorsal nerve roots. Segmental demyelination was not observed in dorsal or ventral roots of either affected or control cats.

Molecular genetic investigations. The genetic, clinical, and histopathologic findings in the SMA cats closely mimicked the type III 5qSMA phenotype of humans. We were prompted, therefore, to determine whether the disorder in affected cats was due to mutation of $S M N$. Feline $S M N$ cDNA was cloned and sequenced. The cDNA had 24 bp of 5' UTR sequence, an 


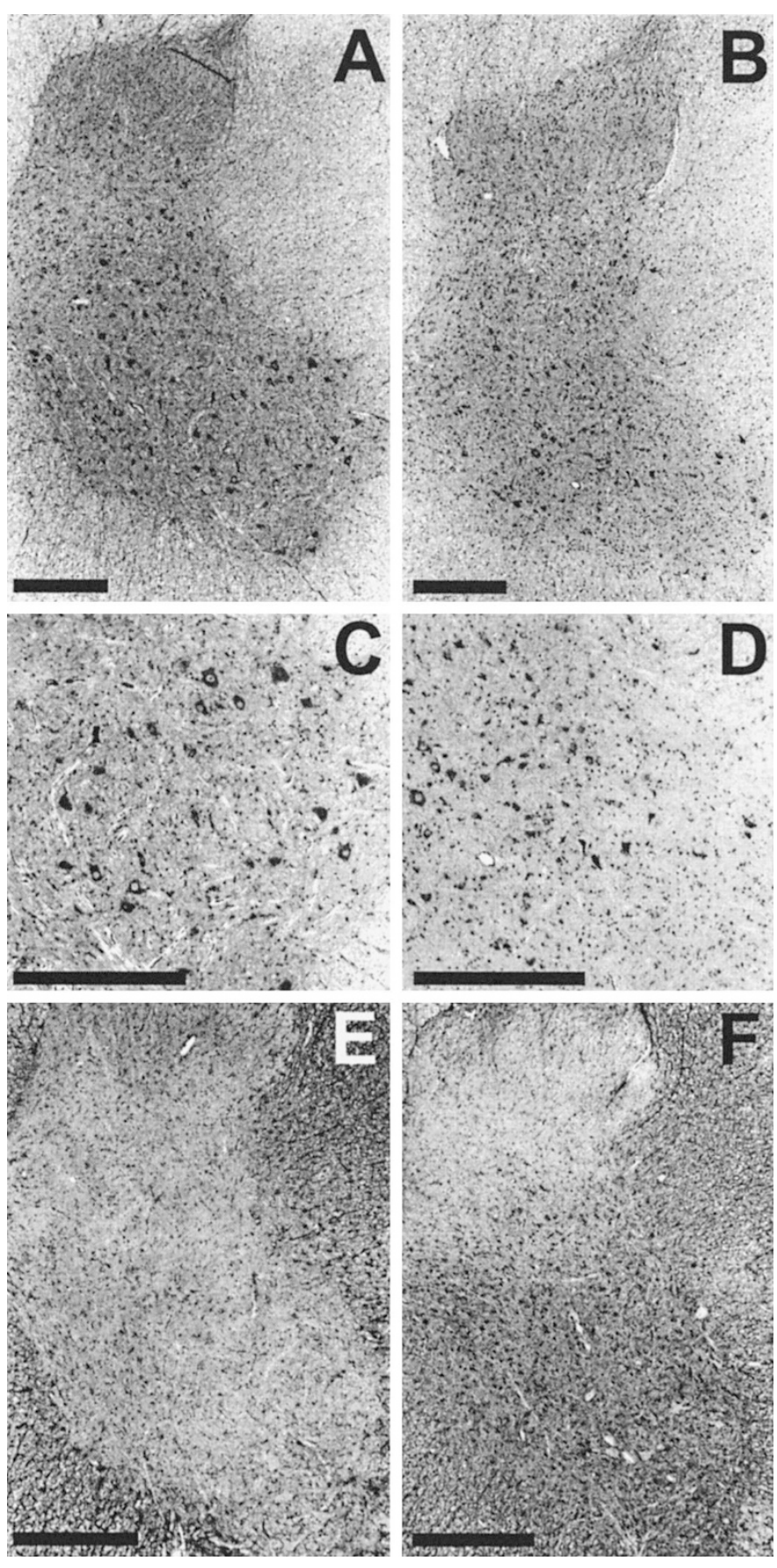

Figure 4. Photomicrographs of spinal cord gray matter of 5.5-mo-old cats. Shown are cresyl violet-stained sections from the sixth cervical segment of a clinically normal cat $(A)$ and a cat exhibiting clinical signs of SMA $(B)$. Ventral horns are toward the bottom of each panel. $(C$ and $D$ ) Magnified images of the ventral horn gray matter from $A$ and $B$, respectively. Also shown are similarly oriented serial sections of the normal $(E)$ and the affected cat $(F)$ spinal cords immunostained for glial fibrillary acidic protein. Bars $=400 \mu \mathrm{m}$.

870-bp open reading frame, and 398 bp of 3' UTR sequence that included a polyadenylation (poly A) signal 15 bp upstream of the poly A tract. The open reading frame encoded a polypeptide of 290 residues, and all of the functional domains defined in human and mouse sequences were conserved, including the exon 7 AG-rich splice enhancer sequence. The feline $S M N$ coding sequence exhibited $94,91,90,87,84$, and $83 \%$ nucleotide identity with canine, human (SMN1), human (SMN2),
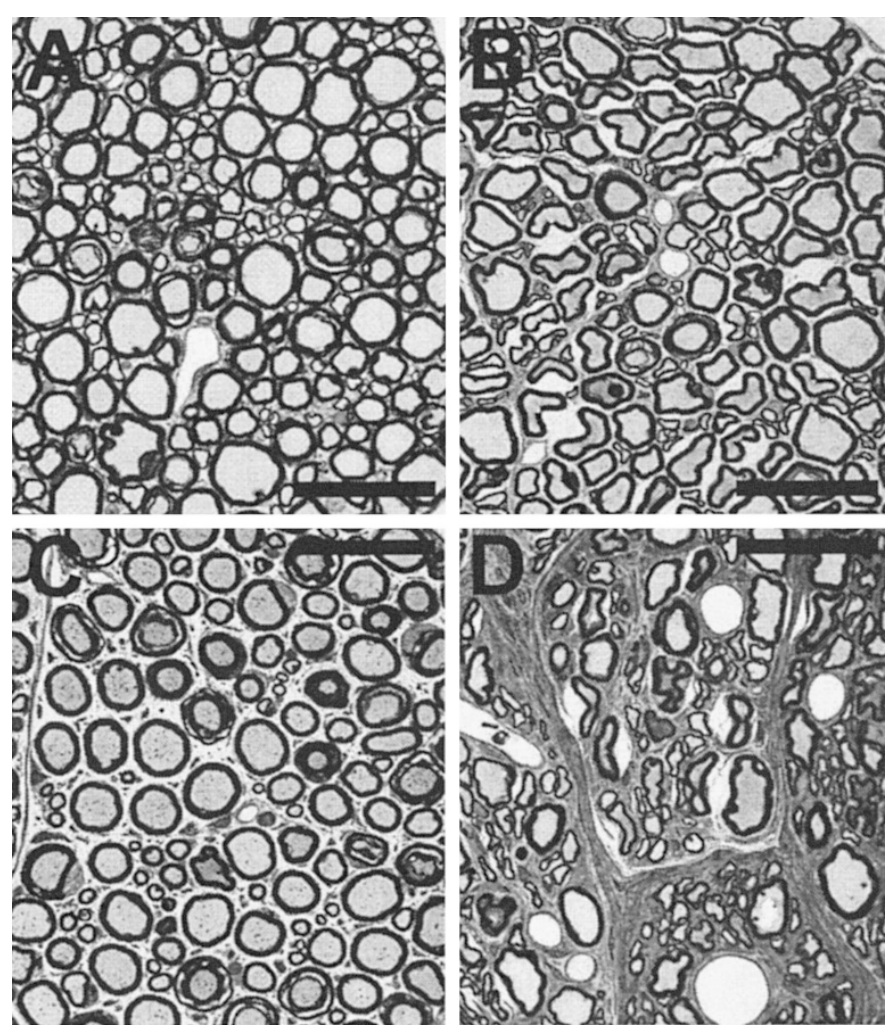

Figure 5. Photomicrographs of cervical spinal nerve roots. Shown are toluidine blue-stained cross-sections of dorsal $(A$ and $B)$ and ventral $(C$ and $D)$ $\mathrm{C}_{5}$ spinal nerve roots of a 5-y-old affected cat $(B$ and $D$ ) and an age-matched control ( $A$ and $C$ ), demonstrating depletion of the large-diameter axon population and extensive endoneurial connective tissue in the affected cat ventral nerve root. No abnormalities were observed in affected cat dorsal roots. Bars $=40 \mu \mathrm{m}$.

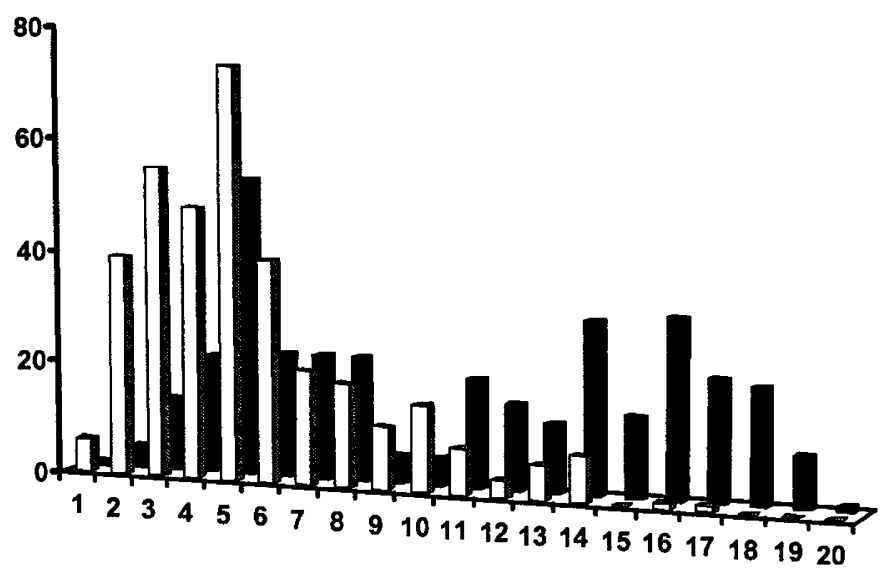

Figure 6. Representative histogram of axon counts and diameters in $\mathrm{C}_{5}$ ventral spinal nerve roots of a 5-y-old affected cat $(\square)$ and an age-matched control (ם). All axons in photographs of randomly selected high-power fields were measured and counted. The $x$ axis indicates axon diameter in microns, and the $y$ axis indicates the number of axons observed of each diameter.

bovine, murine, and rat $S M N$ sequences, respectively, as found in GenBank. Expression analysis by RT-PCR using several primer pairs revealed no evidence of alternative splicing of exon 7, but there was a minor amount of transcript in liver RNA that had a 26-bp deletion in exon 8, apparently as a result of the use of a cryptic polypyrimidine tract and splice acceptor 
site (TTCTTTATTAG). Exon 8 is noncoding, so the alternative splice product does not change the predicted protein product.

In normal feline $S M N$ cDNAs, there were translationally silent sequence variations in exon $3(\mathrm{C} / \mathrm{T})$ and exon $5(\mathrm{~A} / \mathrm{T})$ that led us to consider the possibility that cats might have two expressed $S M N$ genes, as do humans (18). For testing this hypothesis, blots of cat genomic DNA digested with several restriction endonucleases were hybridized to a genomic DNA probe that spanned 150 bp of exon 3 , intron 3 , exon 4 , and 120 bp of intron 4 . The remaining $2.63 \mathrm{kbp}$ of intron 4 was not used in the probe because of the presence of seven copies of a short interspersed repetitive DNA element. In every digest, the $S M N$ probe hybridized to a single band with size varying between 3 and $20 \mathrm{~kb}$, indicating a single $S M N$ locus in the feline genome (Fig. 7). To determine whether the homology of synteny in the $S M N$ region was maintained between cats and humans, we used PCR primers designed from exon 3 and intron 3 to amplify DNA of a feline radiation hybrid panel $(14,19)$. Feline $S M N$ mapped as a single locus on feline chromosome A1 near the genes HEXB and PPAP $2 A$ and $\sim 15 \mathrm{cR}$ from the feline microsatellite FCA084 (data not shown) (16).

RT-PCR amplification and sequencing of SMN cDNA from affected cats revealed no variation from normal in the coding region or in the minor splice variant of the 3' UTR. To address the possibility of an SMN mutation outside of protein coding sequences causing the disorder, we examined genetic linkage of an intragenic $S M N$ marker and the disease phenotype. By sequencing PCR products amplified from genomic DNA of an obligate SMA carrier, a sequence variation of feline $S M N$ was found in intron 4 (four versus five tandem GTTT repeats). Alleles of the SMN marker were assessed in two SMA carriers and nine of their offspring (Fig. 8). The parents were heterozygous (4/5) at the SMN locus, two affected offspring were homozygous $4 / 4$, and four normal offspring were either heterozygous or homozygous $5 / 5$. However, two affected offspring were heterozygous, and one normal offspring was homozygous $4 / 4$, thus indicating at least three recombination events between the SMN marker and the disease locus in the nine offspring. Because the disease locus genotype of the clinically normal offspring was not known, it is possible that additional recombination events were not detected. In the simplest analysis, the hypothesis that $S M N$ is the genetic

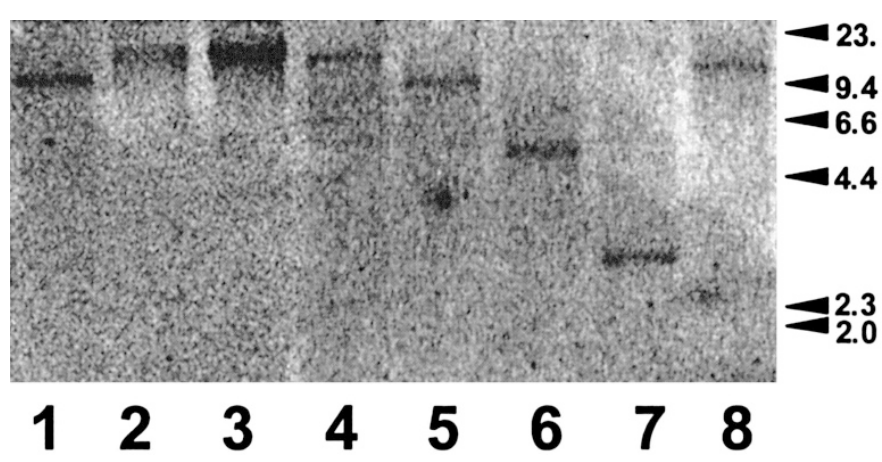

Figure 7. $S M N$ Southern blot of cat genomic DNA. DNA in lanes 1 through 8 was digested with the restriction enzymes SacI, XhoI, NotI, BglII, StuI, XbaI, HindIII, and BamHI, respectively. The SMN probe included exons 3 and 4, intron 3 , and a portion of intron 4 .

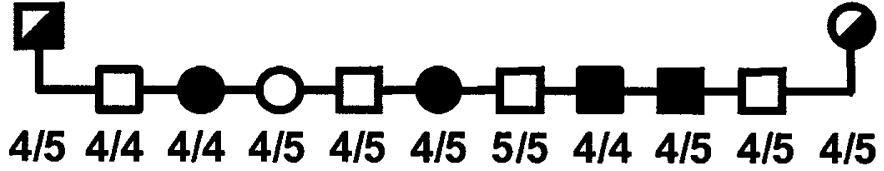

Figure 8. Linkage analysis of an $S M N$ polymorphism in the feline SMA family. The conventions indicating gender and phenotype are described in the Fig. 1 legend. A tandem repeat polymorphism (four $v s$ five repeats of GTTT) in intron 4 of the $S M N$ gene was evaluated in each cat by PCR and gel electrophoresis. Marker alleles of each cat are shown and indicate at least three recombination events between the disease locus and the SMN marker, thus excluding SMN from the disease locus.

locus underlying this fully penetrant, autosomal recessive disease demands that affected animals be homozygous for one $S M N$ allele, obligate carriers be heterozygous, and clinically normal offspring be either heterozygous or homozygous for the SMN allele not seen in affected animals. Therefore, these data indicate that the disease and the $S M N$ loci segregate independently or are only distantly linked and exclude the possibility that a mutation of the single $S M N$ locus of cats causes SMA in this family.

\section{DISCUSSION}

Spinal muscular atrophy was observed in a family of domestic cats. Similar to the human disorder, this feline SMA is a simple autosomal recessive trait that is caused by loss of lower motor neurons of the spinal cord and the consequent neurogenic muscle atrophy. Affected cats exhibited no signs of upper motor neuron or sensory neuron deficit even after several years of disease. Signs of muscle weakness were most prominent in proximal muscles of the hindquarters. Onset of signs occurred at 3-4 mo of age, the initial rapid decline in motor function slowed after some months, and disabled cats survived with varying degrees of disability for many years. Thus, the slowly progressive feline disorder is phenotypically similar to a mild form of SMA in humans and distinct from disorders that involve both upper and lower motor neurons or motor and sensory neurons.

EMG findings and pathologic changes in muscle and peripheral nerve specimens were consistent with muscle denervation. The most salient pathologic changes identified were loss of lower motor neurons of the spinal cord ventral horns and loss of axons in ventral nerve roots. Histopathologic evidence that the degenerative process was confined to lower motor neurons of the ventral horn was 3-fold: dorsal horn nerve roots were not affected; astrogliosis occurred solely in ventral horns; and no lesions were observed in the spinal cord white matter tracts, brainstem, or more rostrally. Examination of spinal cord from affected cats at various ages suggested that the pathologic process was a progressive loss of neurons rather than failure of neurons to develop or migrate properly. However, because affected cats were ascertained by the onset of clinical signs, these studies do not address the question of whether the disease is primarily a retrograde distal axonopathy or first affects the motor neuron somata. Motor neuron disease characterized by a paucity of motor neurons and astrogliosis was previously reported in three unrelated cats but whose clinical signs began after 6 y of age (20). 
Although phenotypically and pathologically similar to human 5qSMA type III, the feline disorder described here is not caused by mutation of the SMN gene. This is, perhaps, not surprising because it seems from human and mouse studies that at least some residual SMN activity is required for embryogenesis $(1,2)$. However, the $S M N$ gene product is a member of a number of cytoplasmic and nuclear protein complexes of incompletely defined constituents and functions $(2,21,22)$. Therefore, SMA cats may have a defect of another member of an SMN complex that mediates an essential function that is lost in 5qSMA patients and, although still unknown, is required for motor neuron survival but not embryogenesis. Obviously, however, although the cat mutation may reside in a gene not as yet implicated in motor neuron disease, it is appropriate to consider as candidates any of the other genes that are already shown to underlie similar human or animal phenotypes.

One such is the immunoglobulin heavy chain $\mu$ binding protein 2 gene (IGHMBP2), mutation of which causes the naturally occurring neuromuscular degeneration $(\mathrm{nmd})$ phenotype of mice and SMA with respiratory distress (SMARD1, OMIM no. 604320) of humans $(23,24)$. However, the feline IGHMBP2 locus was excluded as the feline SMA gene by the same approach of intragenic marker development and linkage analysis demonstrated in this report (Q.H. and J.C.F., unpublished data). Other gene candidates that are implicated by known, naturally occurring mutations in mouse models (e.g. tubulin-specific chaperone e in the progressive motor neuropathy mouse), targeted or random mutagenesis genetic knockouts (e.g. ciliary neurotrophic factor and its receptor), or transgene overexpression (e.g. neurofilaments $\mathrm{L}$ and $\mathrm{M}$ ) are also being investigated (25-27).

In many models of motor neuron degeneration, including the wobbler mouse, a disease interval has been mapped but a disease gene has not yet been determined $(25,28)$. Genes that map to these disease intervals are also considered feline SMA candidates by virtue of their proximity to the disease locus. One such comparative positional candidate gene comes from the recent mapping of bovine SMA to an interval that includes the apoptosis-inhibiting protein encoded by BCL2 (29). Apoptotic pathways are further implicated in motor neuron disease by the recent determination that an antiapoptotic activity of the mitochondrial intermembrane protein, Omi, is abrogated by mutation in the mouse disorder known as motor neuron degeneration 2 (30).

A breeding colony of SMA cats has been established at Michigan State University. Because there are a large number of functional and positional candidate genes nominated by comparative considerations, we plan an unbiased approach to define the feline SMA disease locus by whole-genome scanning. Regardless of the outcome of genetic characterization of the disorder, further development of this SMA model will provide a system in which to investigate mechanisms of neuropathogenesis and potential treatment of motor neuronopathies. Advantages of the domestic cat species in a model of SMA includes that there is a large body of preexisting literature regarding normal development, anatomy, and physiology of the feline CNS. In addition, the relatively large size of cats will allow one to test the safety, effectiveness, and/or practicality of potential diagnostic or therapeutic manipulations before use in human patients.

\section{REFERENCES}

1. Talbot K, Davies KE 2001 Spinal muscular atrophy. Semin Neurol 21:189-197

2. Sendtner M 2001 Molecular mechanisms in spinal muscular atrophy: models and perspectives. Curr Opin Neurol 14:629-634

3. Panozzo C, Frugier T, Cifuentes-Diaz C, Melki J 2001 Spinal muscular atrophy. In: Scriver CR, Beaudet AL, Valle D, Sly WS, Childs B, Kinsler KW, Vogelstein B (eds) The Metabolic and Molecular Bases of Inherited Disease, 8th Ed. McGraw-Hill, New York, pp 5833-5843

4. Lefebvre S, Bürglen L, Frézal J, Munnich A, Melki J 1998 The role of the SMN gene in proximal spinal muscular atrophy. Hum Mol Genet 7:1531-1536

5. Crawford TO 1996 From enigmatic to problematic: the new molecular genetics of childhood spinal muscular atrophy. Neurology 46:335-340

6. Clarke G, Collins RA, Leavitt BR, Andrews DF, Hayden MR, Lumsden CJ, McInnes RR 2000 A one-hit model of cell death in inherited neuronal degenerations. Nature 406:195-199

7. Wong PC, Rothstein JD, Price DL 1998 The genetic and molecular mechanisms of motor neuron disease. Curr Opin Neurobiol 8:791-799

8. Lefebvre S, Bürglen L, Reboullet S, Clermont O, Burlett P, Viollet L, Benichou B, Cruaud C, Millasseau P, Zeviani M, Le Paslier D, Frézal J, Cohen D, Weissenbach J, Munnich A, Melki J 1995 Identification and characterization of a spinal muscular atrophy determining gene. Cell 80:155-165

9. Rudnik-Schoneborn S, Rohrig D, Morgan G, Wirth B, Zerres K 1994 Autosomal recessive proximal spinal muscular atrophy on 101 sibs out of 48 families: clinical picture, influence of gender, and genetic implications. Am J Med Genet 51:70-76

10. Dickinson PJ, LeCouteur RA 2002 Muscle and nerve biopsy. In: Shelton GD (ed) The Veterinary Clinics of North America: Small Animal Practice-Neuromuscular Diseases, Vol 32, No 1. WB Saunders Co., Philadelphia, pp 63-102

11. Cruz-Orive LM 1997 Stereology of single objects. J Microsc 186:93-107

12. Henthorn PS, Somberg RL, Fimiani VM, Puck JM, Patterson DF, Felsburg PJ 1994 IL-2R $\gamma$ gene microdeletion demonstrates that canine $\mathrm{X}$-linked severe combined immunodeficiency is a homologue of the human disease. Genomics 23:69-74

13. Israel DI 1993 A PCR-based method for high stringency screening of DNA libraries. Nucleic Acids Res 21:2627-2631

14. Murphy WJ, Sun S, Chen Z, Yuhki N, Hirschmann D, Menotti-Raymond M, O'Brien SJ 2000 A radiation hybrid map of the cat genome: implications for comparative mapping. Genome Res 10:691-702

15. Sambrook J, Russell DW 2001 Molecular Cloning: A Laboratory Manual, 3rd Ed. Cold Spring Harbor Laboratory Press, Cold Spring Harbor, pp 6.1-6.64

16. Menotti-Raymond M, David VA, Chen ZQ, Menotti KA, Sun S, Schäffer AA, Tomlin JF, Agarwala R, O'Brien SJ, Murphy WJ 2003 Second-generation integrated genetic linkage/radiation hybrid maps of the domestic cat (Felis catus). J Hered 94:95-106

17. Jezyk PF, Haskins ME, Patterson DF 1982 Screening for inborn errors of metabolism in dogs and cats. Prog Clin Biol Res 94:93-116

18. Rochette CF, Gilbert N, Simard LR 2001 SMN gene duplication and the emergence of the $S M N 2$ gene occurred in distinct hominids: SMN2 is unique to Homo sapiens. Hum Genet 108:255-266

19. Sun S, Murphy WJ, Menotti-Raymond M, O'Brien SJ 2001 Integration of the feline radiation hybrid and linkage maps. Mamm Genome 12:436-441

20. Shelton GD, Hopkins AL, Ginn PE, de Lahunta A, Cummings JF, Berryman FC, Hansen L 1998 Adult-onset motor neuron disease in three cats. J Am Vet Med Assoc 212:1271-1275

21. Terns MP, Terns RM 2001 Macromolecular complexes: SMN—the master assembler. Curr Biol 11:R862-R864

22. Rossoll W, Jablonka S, Andreassi C, Kroning AK, Karle K, Monani UR, Sendtner M 2003 Smn, the spinal muscular atrophy-determining gene product, modulates axon growth and localization of beta-actin mRNA in growth cones of motoneurons. J Cell Biol 163:801-812

23. Grohmann K, Schuelke M, Diers A, Hoffmann K, Lucke B, Adams C, Bertini E, Leonhardt-Horti H, Muntoni F, Ouvrier R, Pfeufer A, Rossi R, Van Maldergem L, Wilmshurst JM, Wienker TF, Sendtner M, Rudnik-Schoneborn S, Zerres K, Hubner C 2001 Mutations in the gene encoding immunoglobulin mu-binding protein 2 cause spinal muscular atrophy with respiratory distress type 1 . Nat Genet 29:75-77

24. Cox GA, Mahaffey CL, Frankel WN 1998 Identification of the mouse neuromuscular degeneration gene and mapping of a second site suppressor allele. Neuron 21:13271337

25. Nicholson SJ, Witherden AS, Hafezparast M, Martin JE, Fisher EM 2000 Mice, the motor system, and human motor neuron pathology. Mamm Genome 11:1041-1052

26. Jablonka S, Wiese S, Sendtner M 2004 Axonal defects in mouse models of motoneuron disease. J Neurobiol 58:272-286

27. Martin N, Jaubert J, Gounon P, Salido E, Haase G, Szatanik M, Guénet J-L 2002 A missense mutation in Tbce causes progressive motor neuronopathy in mice. Nat Genet 32:443-447

28. Fuchs S, Resch K, Thiel C, Ulbrich M, Platzer M, Jockusch H, Schmitt-John T 2002 Comparative transcription map of the wobbler critical region of mouse chromosome 11 and the homologous region on human chromosome 2p13-14. BMC Genet 3:14

29. Medugorac I, Kemter J, Russ I, Pietrowski D, Nuske S, Reichenbach HD, Schmahl W, Forster M 2003 Mapping of the bovine spinal muscular atrophy locus to Chromosome 24. Mamm Genome 14:383-391

30. Jones JM, Datta P, Srinivasula SM, Ji W, Gupta S, Zhang Z, Davies E, Hajnoczky G, Saunders TL, Van Keuren ML, Fernandes-Alnemri T, Meisler MH, Alnemri ES 2003 Loss of Omi mitochondrial protease activity causes the neuromuscular disorder of motor neuron degeneration 2 mutant mice. Nature 425:721-727 